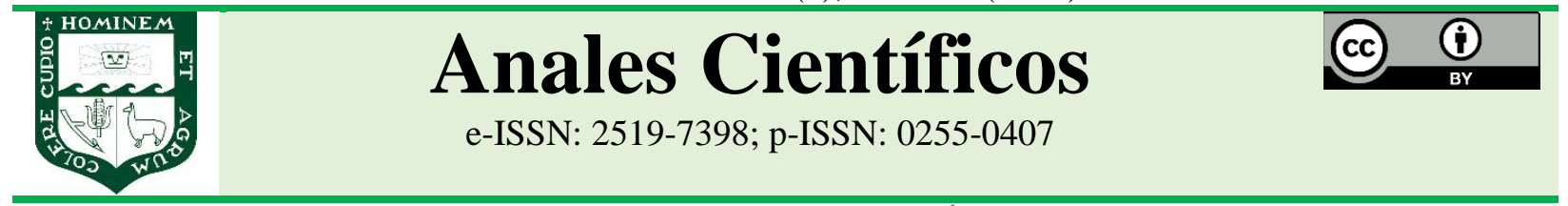

ARTÍCULO ORIGINAL - RESEARCH ARTICLE

http://dx.doi.org/10.21704/ac.v82i2.1784

\title{
EL COMPORTAMIENTO HIGIÉNICO DE Apis mellifera EN RELACIÓN CON EL NIVEL DE INFESTACIÓN DE Varroa destructor
}

\section{The hygienic behaviour of Apis mellifera related to the level of infestation of Varroa destructor}

\author{
Amalia Paco' ${ }^{1}$; ; Agustín Martos²*iD; Víctor López ${ }^{3}$; Julián Chura ${ }^{4}$ (DD \\ ${ }^{1}$ Facultad de Ciencias Agrarias, Universidad Nacional de San Antonio Abad del Cusco, Perú \\ ${ }^{2}$ Departamento de Entomología, Universidad Nacional Agraria La Molina, Lima, Perú \\ ${ }^{3}$ Facultad de Ciencias Agrarias, Universidad Nacional De San Antonio Abad del Cusco, Perú \\ ${ }^{4}$ Departamento de Fitotecnia, Universidad Nacional Agraria La Molina, Lima, Perú \\ *E-mail: amartos@lamolina.edu.pe
}

Recibido: 02/01/2021; Aceptado: 01/11/2021; Publicado: 31/12/2021

\begin{abstract}
Hygienic behavior and the infestation level of varroa on adult bees, and the relation between those variables, were assessed on Apis mellifera bee colonies. The hygienic behavior was determined through the pin-killed test and the infestation level of varroa on adult bees was calculated on adult bee samples through the sieve technique. Correlation test between hygienic behavior and infestation level of varroa on adult bees was determined using the Spearman correlation test and SAS program 9.4 version. The hygienic behavior average value was $99,8 \%$ for the $52 \%$ highly hygienic bee colonies, while the infestation level of varroa on adult bees average value was $1,69 \%$ for the $92,3 \%$ low mite infestation colonies. Negative correlation $\left(r_{s}=0,397\right)$ highly significant $(p<0,01)$ was found. Results found suggest the existence of A. mellifera colonies with Varroa destructor resistant characteristics, due to high values of hygienic behavior and low infestation levels of varroa on adult bees.
\end{abstract}

Keywords. Apis mellifera | Varroa destructor | Spearman correlation test | varroasis

\section{RESUMEN}

El comportamiento higiénico y el nivel de infestación de varroa sobre abejas adultas, y la relación entre estas variables, fueron evaluadas sobre colonias de abejas Apis mellifera. El comportamiento higiénico se determinó mediante la técnica del pinchando de pupas con aguja, y la tasa de infestación de varroa en abejas adultas se calculó en muestras de abejas adultas por el método del tamizado. La correlación entre el comportamiento higiénico y la tasa de infestación de varroa en abejas adultas fue determinado usando la prueba de correlación de Spearman y el programa SAS versión 9.4. El valor promedio de comportamiento higiénico fue de 99,8\% para el 52\% de colonias altamente higiénicas, mientras que la tasa de infestación de varroa en abejas adultas promedio fue de 1,69\% para el 92,3\% de colonias con baja infestación por el ácaro. Se encontró una correlación negativa $\left(\mathrm{r}_{\mathrm{s}}=-0,397\right)$ altamente significativa $(\mathrm{P}<0,01)$. Los resultados encontrados sugieren la existencia de colonias de A. mellifera con 
características de resistencia a Varroa destructor, por los altos valores de comportamiento higiénico y las bajas tasas de infestación de varroa en abejas adultas.

Palabras clave: Apis mellifera | Varroa destructor | prueba de correlación de Spearman | varroasis

Forma de citar el artículo (Formato APA):

Paco, A., Martos, A., López, V., \& Chura, J. (2021). El comportamiento higiénico de Apis mellifera en relación al nivel de infestación de Varroa destructor. Anales Científicos. 81(2), 219-226. http://dx.doi.org/10.21704/ac.v82i2.1784

Autor de correspondencia (*): Agustín Martos. Email: amartos@lamolina.edu.pe

(C) Los autores. Publicado por la Universidad Nacional Agraria La Molina.

This is an open access article under the CC BY

\section{INTRODUCCIÓN}

Apis mellifera L. o abeja melífera es una especie de gran importancia económica por producir miel, otros productos de la colmena y alimentos diversos gracias a su importante papel como agente polinizador (Chmiel et al., 2020). Los especialistas en apicultura la venimos utilizando en el Perú en empresas de agro exportación como agente polinizador en diversos cultivos frutales como el palto y arándanos. Se ha observado que la abeja melífera durante su desarrollo es atacada por diversas plagas y enfermedades, siendo la varroasis, causada por el ácaro Varroa destructor, y la loque europea, ocasionada por la bacteria Melissococcus pluton, los problemas sanitarios más importantes (Montenegro, 2016). Sin embargo, la abeja melífera exhibe mecanismos que podrían estar relacionados a una resistencia natural como el comportamiento higiénico por medio del cual retiran las pupas enfermas, parasitadas o muertas presentes en las celdas de los panales de cría, acción que no escapa a nuestra observación durante la revisión de las colmenas.

De Jong (2010) refiere que las sustancias químicas que se emplean para el control de plagas en abejas melíferas contaminan la miel y otros productos de la colmena, generan resistencia en las plagas, afectan negativamente la resistencia natural y ocasionan gastos económicos. Sin embargo, las abejas han desarrollado mecanismos de defensa contra problemas sanitarios, entre los que se tiene el comportamiento higiénico, el cual se define como la habilidad que tienen las abejas obreras para detectar, desopercular y remover las crías enfermas y muertas hacia el exterior de la colonia (Principal et al, 2008), lo cual constituye un mecanismo primario de resistencia ante problemas sanitarios como la varroasis (Guerra et al. (2000), citados por Principal et al. (2008). Marcangeli (1997) sostiene que el comportamiento higiénico puede ser herramienta útil para la selección y multiplicación de líneas de abejas menos susceptibles a varroa; en abejas híbridas A. mellifera mellifera x A. mellifera ligústica, encontró que las colonias con mayor comportamiento higiénico presentaron menores porcentajes o tasas de infestación por la varroa en abejas adultas, lo cual se traduce en una correlación negativa significativa entre el comportamiento higiénico y la infestación del ácaro, (r= $-0.749, \mathrm{p}<0.01$ ). Gramacho y Spivak (2003) afirman que las abejas con mayor sensibilidad olfatoria son capaces de detectar y discriminar con precisión crías normales y enfermas, siendo el comportamiento higiénico una característica genética. Gramacho (2004) indica que en la selección genética de abejas se busca obtener líneas con buenas características productivas y, principalmente, resistentes a plagas y enfermedades. Marcangeli (2004) encontró que las colmenas vigorosas tuvieron porcentajes de remoción significativamente más altos que las debilitadas, con valores de $74,3 \%$ y $57,6 \%$, respectivamente. Ivernizzi y Rodríguez (2007) encontraron un incremento de $77,7 \%$ a $98,7 \%$ en el comportamiento higiénico con reducción significativa en los niveles de enfermedades de crías y varroasis, después de seis generaciones, en el marco de un trabajo de mejoramiento de abejas melíferas.

Principal et al. (2008), en apiarios con abejas africanizadas en Venezuela, determinaron colmenas en las categorías de levemente higiénicas (17\%), moderadamente higiénicas (24\%) y altamente higiénicas (59\%); los valores de comportamiento higiénico registrados fueron menor del $75 \%$ en la primera categoría, en la segunda categoría registraron valores de 75 a $95 \%$, y en la tercera categoría tuvieron valores mayores a 95\%. De Jong (2010) indica que criando reinas de colmenas sanas a través de generaciones se puede seleccionar abejas resistentes a enfermedades, en tanto que Arechavaleta et al. (2011) afirman que el 
comportamiento higiénico es una característica genética importante en abejas melíferas para fines de control de patógenos y parásitos; lo cual es apoyado por Jiang et al. (2016) quienes afirman que la tolerancia a varroa es genética. Pinto et al. (2012), en dos colmenares en Brasil, uno en Taubaté y otro en Viçosa, en abejas africanizadas A. mellifera, encontraron correlación negativa entre el porcentaje de infestación del ácaro $V$. destructor y el comportamiento higiénico de las abejas $(\mathrm{r}=-0.9627$; $\mathrm{p}<0.01)$; en Taubaté el valor promedio de la tasa de infestación por el ácaro fue de 4,9\% (3,4 a 5,8\%) con un comportamiento higiénico de $98,6 \%$ (96 a 100\%), mientras que en Viçosa la tasa de infestación promedio de la plaga fue de $10 \%(5,4-21 \%)$ con un comportamiento higiénico de $57,7 \%$ (0-79\%), lo cual sugiere que las colonias con comportamiento higiénico elevado poseen menores tasas de infestación. De Jong (2014) refiere que, en colmenas con reina joven y de buena postura, el comportamiento higiénico es más intenso y eficiente, situación que no se observa en colonias con reina vieja y de escasa postura. Medina et al. (2014) encontraron que colonias con comportamiento higiénico alto produjeron significativamente más miel que las de comportamiento higiénico bajo, en el marco de infestaciones similares de varroa entre grupos de colonias. Ariclenes et al. (2015), en Paraíba-Brasil determinaron que la mayoría de colmenas evaluadas fueron consideradas higiénicas con índices superiores a $80 \%$ de comportamiento higiénico.

Castagnino et al. (2016) afirman que el comportamiento higiénico en A. mellifera es uno de los más importantes mecanismos de defensa contra las enfermedades de las crías de las abejas; refieren, además, que colmenas con valores de 82,5 \% de comportamiento higiénico, categorizadas como colonias higiénicas, tienen potencial genético para producción de reinas seleccionadas; demostraron, además, en colmenas africanizadas de la UFBA-Brasil, una correlación negativa entre el comportamiento higiénico $(82,5 \%)$ que corresponde a colonias higiénicas con una infestación por $V$. destructor en abejas adultas $(4,19 \%)$, lo cual indica que colonias con mayor comportamiento higiénico tuvieron menores tasas de infestación de varroa. Pires et al. (2006), Pérez y Demedio (2014) y Espinoza et al. (2008) demostraron la eficacia de la técnica de punción con un alfiler entomológico en la evaluación del comportamiento higiénico en abejas melíferas; realizaron el pinchado de 100 pupas frescas y después de 24 horas efectuaron la lectura del número de celdas limpias o vacías. Marcangeli y Damiani (2007) determinaron mayor infestación por varroa en panales viejos que en nuevos con registros de $13,5 \%$ y $6,2 \%$, respectivamente, infestación que se vería favorecida por los olores atrayentes emanados de las celdas. Sanabria et al. (2015) encontraron mayores tasas de infestación de varroa en abejas adultas en colmenas con tratamiento químico $(5,36)$ respecto a colmenas sin tratamiento $(3,61 \%)$. Vandame (2001), para determinar el porcentaje de infestación de varroa en abejas adultas, propone emplear el método de David De Jong o del tamizado, el cual consiste en colocar una muestra de abejas en solución de agua con detergente, tamizarlas y contar el número de ácaros y abejas; luego, mediante una regla de tres simple se puede obtener el porcentaje de infestación donde el número de abejas es el $100 \%$.

La presente investigación se planteó con el objetivo de estudiar la relación entre el comportamiento higiénico y la tasa de infestación de varroa en abejas adultas.

\section{MATERIALES Y MÉTODOS}

La investigación se desarrolló en el apiario de la Universidad Nacional Agraria La Molina (UNALM) sobre 52 colonias dóciles de abejas A. mellifera con manejo y condiciones similares, sin aplicaciones químicas para el control de plagas y enfermedades, y en cámara de cría con alrededor de 20,000 abeja adultas y panales de cría y reserva alimenticia; entre finales de verano e inicios de otoño, época de clima agradable para las abejas.

Para la determinación del comportamiento higiénico o porcentaje de remoción de pupas pinchadas se siguió la técnica del pinchado de pupas (Pires et al. 2006, Pérez y Demedio, 2014 y Espinoza et al., 2008). Se tomó de cada colonia un panal con cría operculada de obrera con pupas con ojos de color rosado; en la parte central del panal se delimitó con mondadientes un área con 100 crías operculadas de obrera y, una por una, fueron pinchadas con aguja de jeringa de inyectable; el panal, marcado en el cabezal, fue reacondicionado en la colmena y luego de 24 horas se procedió a la lectura del número de pupas removidas completamente. Con estos datos se calculó el porcentaje de pupas removidas o porcentaje de comportamiento higiénico con una 
fórmula en base a la regla de tres simple: \% de pupas removidas o Comportamiento Higiénico = número de pupas removidas x 100/número de pupas pinchas. Los valores de comportamiento higiénico, siguiendo el criterio de Spivak (1996), citado por Principal et al. (2008), permitieron categorizar las colmenas en: Altamente Higiénicas (>95\% de pupas removidas), Moderadamente Higiénicas (75 a 95\% de pupas removidas) y Levemente Higiénicas $(<75 \%$ de pupas removidas).

El porcentaje o tasa de infestación de varroa en abejas adultas se hizo sobre una muestra de aproximadamente 300 abejas a través de la técnica del tamizado (Vandame, 2001). Para ello, se extrajo de cada colmena un panal de cría con abejas y se sacudió sobre una bandeja de fondo blanco con agua con detergente; el medio líquido fue agitado y pasado por un tamiz para separar las varroas del cuerpo de las abejas, ayudado por varios enjuagues; las varroas pasaron al medio líquido de donde fueron retiradas y contadas; las abejas también fueron contadas. Con estos datos se calculó el porcentaje o tasa de infestación de varroa en abejas adultas mediante una fórmula en base a la regla de tres simple: Tasa de infestación de varroa en abejas adultas = número de varroas x 100/número de abejas. Los valores de tasa de infestación de varroa en abejas adultas sirvieron para categorizar colmenas, siguiendo el criterio de Vandame (2001), en tres categorías de infestación: Bajo (0-5\%), Moderado (5.1 a 10\%) y Alto (>10\%).

La correlación entre los valores de comportamiento higiénico y la tasa o porcentaje de infestación de varroa en abejas adultas se analizó a través del coeficiente de correlación de Spearman $\left(\mathrm{r}_{\mathrm{s}}\right)$, utilizando el programa SAS (Statistical Analysis System) versión 9.4.

\section{RESULTADOS Y DISCUSIÓN}

Los valores de comportamiento higiénico en colmenas fluctuaron entre 20 a $100 \%$, estando la mayoría de los registros bastante cercanos al $100 \%$, con colonias categorizadas como altamente higiénicas (52\%), moderadamente higiénicas (27\%) y levemente higiénicas (21\%). Las abejas demostraron gran habilidad para extraer las pupas muertas, limpiar completamente las celdas y eliminar los individuos fuera de la colmena, lo cual realizan a través de vuelos cortos.

Tabla 1. Categorías de colmenas según porcentajes de comportamiento higiénico.

\begin{tabular}{lcc}
\hline \multicolumn{1}{c}{ Categorías } & $\begin{array}{c}\text { \# y \% de } \\
\text { colmenas } \\
\text { evaluadas }\end{array}$ & $\begin{array}{c}\text { Porcentaje } \\
\text { promedio de } \\
\text { comportamiento } \\
\text { higiénico }\end{array}$ \\
\hline Altamente higiénica & $27 ; 52$ & $99,8[96,0-100]$ \\
\hline $\begin{array}{l}\text { Moderadamente } \\
\text { higiénica }\end{array}$ & $14 ; 27$ & $87,5[75,0-95,0]$ \\
\hline Levemente higiénica & $11 ; 21$ & $47,5[20,0-60,0]$ \\
\hline & $52 ; 100$ & \\
\hline
\end{tabular}

Categorías de colmenas según el porcentaje de comportamiento higiénico [Spivak (1996), citado por Principal et al. (2008)]: Altamente higiénica (De 96 a $100 \%$ ), Moderadamente higiénica (De 75 a $95 \%$ ) y Levemente higiénica (De 0 a $74 \%$ ).

El elevado porcentaje de colonias con alta capacidad higiénica en el apiario de la UNALM sugiere la existencia de valioso patrimonio genético de abejas resistentes a varroa que no requieren de aplicaciones químicas para el manejo de este y otros problemas sanitarios. Por ello, constituyen material genético de gran importancia para fines de selección y propagación de reinas mejoradas genéticamente desde el punto de vista del comportamiento higiénico. En este sentido, a partir de larvas de obrera de estas colonias se sugiere promover la crianza artificial de reinas o realizar la división y multiplicación de colonias a fin de conservar y mejorar las características genéticas favorables en torno al comportamiento higiénico. Investigadores en el mundo han encontrado registros promedios de comportamiento higiénico parecidos a los encontrados en esta investigación, tales como Castagnino et al. (2016) quienes en Brasil encontraron en colmenas un valor promedio de $82,5 \%$ que corresponden a colonias moderadamente higiénicas.

Por su parte, Principal et al. (2008) en Venezuela registraron 59\% de colonias con alta manifestación de comportamiento higiénico con promedios mayores a 95\% de remoción de pupas muertas; mientras que Marcangeli (2004) en colmenas vigorosas y debilitadas encontró tasas de remoción de 74,3\% y 57,6\%, respectivamente, lo cual corresponde a colonias 
levemente higiénicas. También, Ariclenes et al. (2015), en Paraíba-Brasil, determinaron que la mayoría de colmenas evaluadas fueron consideradas higiénicas con índices superiores a $80 \%$ de comportamiento higiénico. La capacidad de remoción de pupas muertas de las celdas por parte de las abejas sugiere un componente genético que varía según colonias de abejas, así como también de un componente ambiental asociado a la calidad de manejo de las colonias. Guerra et al. (2000), citados por Principal et al. (2008), afirman que el comportamiento higiénico constituye un mecanismo primario de resistencia ante problemas sanitarios como la varroasis, mientras que Arechavaleta et al. (2011) manifiestan que es una característica genética importante en abejas melíferas para fines de control de patógenos y parásitos. En este sentido, las abejas con mayor sensibilidad olfatoria son capaces de detectar y discriminar la cría normal de la enferma (Gramacho y Spivak (2003), siendo el comportamiento de limpieza más eficiente en colonias con reina de buena capacidad de postura (De Jong, 2014).

Tabla 2. Categorías de colmenas según la tasa de infestación de varroa en abejas adultas.

\begin{tabular}{lcc}
\hline Categorías & $\begin{array}{c}\text { \# y \% de } \\
\text { colmenas } \\
\text { evaluadas }\end{array}$ & $\begin{array}{c}\text { Tasa promedio de infestación } \\
\text { de varroa en abejas adultas }(\%)\end{array}$ \\
\hline Bajo & $48 ; 92,3$ & $1,69[0-4,93]$ \\
Moderado & $4 ; 7,7$ & $5,59[5,06-6,67]$ \\
Alto & $0 ; 0$ & -- \\
& $52 ; 100$ & \\
\hline
\end{tabular}

Categorías de colmenas según la tasa de infestación de varroa en abejas adultas (Vandame, 2001): Bajo (de 0 a $5 \%$ ), Moderado (de 5,1 a $10 \%$ ) y Alto (> $10 \%$ ).

Marcangeli (1997), por su parte, sostiene que el comportamiento higiénico puede ser herramienta útil para la selección y multiplicación de líneas de abejas menos susceptibles al ácaro causante de la varroasis. Castagnino et al. (2016) afirman que el comportamiento higiénico en A. mellifera es uno de los más importantes mecanismos de defensa contra las enfermedades de las crías de las abejas; refiriendo, además, que colmenas con valores de $82,5 \%$ de comportamiento higiénico, categorizadas como colonias higiénicas, tienen potencial genético para producción de reinas seleccionadas. Abejas con altos valores de comportamiento higiénico pueden ser más productivas, tal como lo determinaron Medina et al. (2014) quienes encontraron que colonias con comportamiento higiénico alto produjeron significativamente más miel que las de comportamiento higiénico bajo.

Por otro lado, los valores de tasa de infestación de varroa en abejas adultas variaron entre 0 a $6,67 \%$, estando la mayoría de los registros cercanos al menor valor, lo cual indica que la gran mayoría de colmenas mostraron bajas infestaciones. La gran mayoría de colonias $(92,3 \%)$ correspondieron a la categoría de infestación baja y unas pocas $(7,7 \%)$ a la categoría de infestación moderada. Una baja infestación por varroa en abejas adultas sugiere que la colonia tiene mecanismos para resistir los embates de la plaga, impidiéndole alcanzar niveles que puedan ocasionar daños importantes en la vida de las abejas y pérdidas económicas en productos de la colmena. Además, indica que los daños por varroa en crías son mínimos y por debajo de daños biológicos y económicos, quedando abierta la posibilidad de contar con material biológico de buena calidad genética para fines de mejoramiento. Esto se sostiene en lo manifestado por Jiang et al. (2016) quienes afirman que la tolerancia de las abejas a varroa es genético, y por De Jong (2010) quienes indican que criando reinas de colmenas sanas a través de generaciones se puede seleccionar abejas resistentes a enfermedades.

Por otra parte, los resultados encontrados en el presente estudio son similares a los encontrados por Sanabria et al. (2015) quienes encontraron valores tasa de infestación de varroa en abejas adultas de 3,61\% en colonias de abejas melíferas sin tratamiento químico, y lo determinado por Pinto et al. (2012) con un porcentaje de 4,9\% en Taubaté-Brasil, sin embargo, contrasta con lo hallado por los mismos autores en Vicosa donde la tasa de infestación de varroa en abejas adultas fluctuó entre 5,4 y $21 \%$. En esta tendencia se encuentra lo registrado por Marcangeli y Damiani (2007), quienes presentan infestaciones entre 6,18 y $13,52 \%$ en panales nuevos y viejos, respectivamente.

Al realizar el análisis de correlación de Spearman entre la tasa (\%) de pupas removidas o comportamiento higiénico y la tasa (\%) de infestación de varroa en abejas adultas se encontró un valor de $\mathrm{r}_{\mathrm{s}}=-0,397$ altamente significativo $\mathrm{p}<0,01$. El valor negativo en el análisis de correlación indica que cuando una variable 
se incrementa, la otra disminuye; en otras palabras, cuando la tasa $(\%)$ de pupas removidas o comportamiento higiénico se incrementa, la tasa $(\%)$ de infestación de varroa en abejas adultas disminuye. Este resultado concuerda con el de Pinto et al. (2012) quienes encontraron correlación negativa $(r=-0.9627$; $\mathrm{p}<0.01)$ entre la la tasa $(\%)$ de infestación de varroa en abejas adultas y el comportamiento higiénico en Taubaté y Vicosa; en el primer lugar geográfico el valor promedio de la tasa de infestación fue de 4,9\% con un comportamiento higiénico promedio de $98.6 \%$, mientras que en el segundo lugar la tasa de infestación promedio fue de $10 \%$ con un comportamiento higiénico promedio de $57.7 \%$. Correlación negativa también fue determinada por Marcangeli (1997) en abejas europeas $(\mathrm{r}=-0.749 ; \mathrm{p}<0.01)$, Arechavaleta et al. (2010) $(\mathrm{r}=$ 0.15: p<0.05) y Castagnino et al. (2016) en abejas africanizadas en Brasil con un comportamiento higiénico de $82,5 \%$ y una la tasa $(\%)$ de infestación de varroa en abejas adultas de $4,19 \%$.

En este mismo sentido y reafirmando lo encontrado en la investigación, se tiene los aportes de Ivernizzi y Rodriguez (2007), quienes indican que el comportamiento higiénico se incrementó en cinco años de $77,7 \%$ a $98,7 \%$, lo cual permite afirmar que puede haber una disminución drástica de las enfermedades a mediano plazo en el marco de un programa de selección masiva. Sin embargo, algunos autores muestran resultados que contrastan con lo encontrado en este estudio, como Araneda et al. (2008) quienes demostraron que no hay correlación entre el nivel de infestación del ácaro con el comportamiento higiénico o tasa $(\%)$ de remoción de pupas porque encontraron valores muy variables entre sí. También, Aumeier et al. (2000) afirman que el comportamiento higiénico no es un factor clave en la tolerancia a varroasis de las abejas africanizadas en Brasil.

Los resultados obtenidos en el presente estudio sugieren la existencia de colonias de $A$. mellifera con características de resistencia a $V$. destructor.

\section{CONCLUSIONES}

Las gran mayoría de colonias de Apis mellifera evaluadas demostraron ser altamente higiénicas y con baja tasa de infestación de Varroa destructor en abejas adultas, con una correlación inversa entre el comportamiento higiénico y la tasa de infestación por varroa, lo cual pone de manifiesto la existencia de colonias de abejas con características genéticas de resistencia a varroa para fines de mejoramiento genético.

\section{Conflictos de intereses}

Los autores firmantes del presente trabajo de investigación declaran no tener ningún potencial conflicto de interés personal o económico con otras personas $\mathrm{u}$ organizaciones que puedan influir indebidamente con el presente manuscrito.

\section{Contribuciones de los autores}

Preparación y ejecución: AP, AM, VL, JC; Desarrollo de la metodología: AP, AM, VL, JC; Concepción y diseño: AP, AM, VL, JC; Edición del artículo: AP, AM, VL, JC; Supervisión del estudio: AP, AM, JC.

\section{REFERENCIAS}

- Araneda, D., Pérez, N., Castillo, R. et al. (2008). Evaluación del comportamiento higiénico de Apis mellifera L. en relación al nivel de infestación de varroa destructor Anderson \& Truenan. IDESIA, 26 (2), 59-67. Disponible en: https://scielo.conicyt.cl/pdf/idesia/v26n2/art08.pdf

- Arechavaleta, M., Robles, C., Straffon, S. et al. (2010). Heredabilidad y correlaciones para la resistencia de las colonias de abejas al crecimiento poblacional de Varroa destructor A. $17^{\circ}$ congreso internacional de actualización apícola, mayo del 2010. Villahermosa, Tabasco, México. Asociación Nacional de Médicos veterinarios especialistas en abejas. A.C.P. p. 88-91.

- Arechavaleta, M., Hunt, G., Spivak, M. et al. (2011). Loci de rasgos binarios que influyen en la expresión del comportamiento higiénico de las abejas melíferas. Rev. Mex. Cienc. Pecu, 2(3), 283$298 . \quad$ Disponible en: http://www.scielo.org.mx/pdf/rmcp/v2n3/v2n3a4.p df.

- Ariclenes, F., Casimiro, D., Carbalho, D., et al. (2015). Compotamento higiênico en colmeias de Apis mellifera L. africanizadas no sertão de Paraíba. Rev. Verde (Pombal-PB-Brasil), 10(3), 08-12. Disponible en: http://oaji.net/articles/2016/22381481128973.pdf 
- Aumeier, P., Rosenkranz, P., \& Gonçalves, L. (2000). A comparison of the hygienic response of Africanized and European (Apis mellifera carnica) honey bees to Varroa-infested brood in tropical Brazil. Genetics and Molecular Biology, 23(4), 787791.

- Castagnino, G., Pinto, L., \& Carneiro, M. (2016). Correlação da infestação de Varroa destructor sobre o comportamento higiênico de abelhas Apis mellifera. Archivos de Zootecnia, 65, 549-554. Disponible

en: https://www.semanticscholar.org/paper/Correla\%C 3\%A7\%C3\%A3o-da-infesta\%C3\%A7\%C3\%A3ode-Varroa-destructor-sobre-Castagnino-

Pinto/4d3cb1d0bd1cb6e0075757bfb825e352e5b1f $2 \mathrm{~cd}$

- Chmiel, J., Daisley, B., Pitek, A., Thompsom, G. \& Reid, G. (2020). Understanding the effects of sublethal pesticide exposure on honey bees: A role for probiotics as mediators of environmental stress. Frontiers in Ecology and Evolution, 8, 1-19 (artículo 22). Disponible en: https://www.frontiersin.org/articles/10.3389/fevo.2 020.00022/full

- De Jong, D. (2010). Nuevas enfermedades de la abejas- selección y manejo para su control. $17^{\circ}$ Congreso Internacional de Actualización Apícola, mayo de 2010. Villahermosa, Tabasco, México. Asociación Nacional de Médicos Veterinarios Especialistas en Abejas. A.C.P. p. 78-80. Disponible en: http://docplayer.es/7456988-17congreso-internacional-de-actualizacion-apicolaasociacion-nacional-de-medicos-veterinariosespecialistas-en-abejas-a-c.html

- De Jong, D. (2014). Selección de abejas para el control de enfermedades especialmente la varroasis.

- Espinoza, L., Guzmán, N., Sánchez A., et al. (2008). Estudio comparativo de tres pruebas para evaluar el comportamiento higiénico en colmenas de abejas (Apis mellifera L.) Vet. Méx., 39, 39-54. Disponible en:

http://www.scielo.org.mx/pdf/vetmex/v39n1/v39n1 a4.pdf

- Gramacho, K. (2004). XV Congreso Brasileiro de Apicultura, $1^{\circ}$ Congreso Brasileiro de Meliponicultura. Brasil.
- Gramacho, K., \& Spivak, M. (2003). Differences in olfactory sensitivity and behavioral responses among honey bees bred for hygienic behavior. Behavioral Ecology and sociobiology, 54(5), 472$479 . \quad$ Disponible en: https://experts.umn.edu/en/publications/differences -in-olfactory-sensitivity-and-behavioral-responsesamo

- Ivernizzi, C., \& Rodríguez, J. (2007). Mejora en la sanidad de la cría en colonias de abejas (Apis mellifera L.) seleccionadas por el comportamiento higiénico. Veterinaria, 42(167), 9-13. Montevideo. Disponible en: https://www.cabi.org/isc/FullTextPDF/2008/20083 223138.pdf

- Jiang, S., Robertson, T., Mostajeran, M. et al. (2016). Differential gene expression of two extreme honey bee (Apis mellifera) colonies showing varroa tolerance and susceptibility. Department of Food and Bioproduct Sciences University of Saskatchewan, Saskatoon, SK, Canada; and Meadow Ridge Enterprises Ltd, Saskatoon, SK, Canada. Insect Molecular Biology, 25(3), 272-282. Disponible en: https://pubmed.ncbi.nlm.nih.gov/26919127/

- Marcangeli, J. (1997). Relación entre el comportamiento higiénico de la abeja Apis mellifera (Hymenoptera: Apidae) y el tamaño poblacional del acaro varroa jacobsoni (Mesostigmata: Varroidae). Natura Neotropicalis, 28(2), 125-129.

- Marcangeli, J. (2004). Factores que influyen sobre la manifestación del comportamiento higiénico de las abejas Apis mellifera (Himenoptera:Apidae). II. Vigor de la colmena. Natura Neotropicalis, 34 y 35, 15-18.

- Marcangeli, J. \& Damiani, N. (2007). Índices de prevalencia del ácaro Varroa destructor (Acari:Varroidae) en cuadros de crías nuevos o previamente utilizados por Apis mellifera (Hymenoptera:Apidae) Rev. Soc. Entomol. Argentina, 66(1-2), 147-152. Disponible en: file:///C:/Users/Agustin/Downloads/3_Indices_Pre valencia.pdf

- Medina, C., Guzmán, E., Aréchida, C. et al. (2014). Producción de miel e infestación con Varroa destructor de abejas africanizadas (Apis mellifera) con alto y bajo comportamiento higiénico. Rev. Mex. Cienc. Реси., 5(2), 157-170. Disponible en: 
http://www.scielo.org.mx/pdf/rmcp/v5n2/v5n2a3.pd f

- Montenegro, G. (2016). Manual Apícola. Pontificia Universidad Católica de Chile. INDAP-Ministerio de Agricultura. 116 pp. Disponible en: https://agronomia.uc.cl/extension/publicaciones1/151-manual-apicola-indap-uc/file

- Pérez, H., \& Demedio, J. (2014). Evaluación de la conducta higiénica en colmenas de abejas Apis mellifera $L$. por el método del pinchado con dos instrumentos. Rev. Salud Animal, 36(3), 170-177. Disponible en: http://scielo.sld.cu/pdf/rsa/v36n3/rsa06314.pdf

- Pinto, F., Puker A., Barreto, L. et al. (2012). The ectoparasite mite Varroa destructor Anderson and Trueman in southeastern Brazil apiaries: effects of the hygienic behavior of africanized honey bees on infestation rates. Arq. Bras. Med. Vet. Zootec., 64(5), 1194-1199. Disponible en http://www.scielo.br/pdf/abmvz/v64n5/v64n5a17.p df
- Pires, S., Josa, A., Martins, A. et al. (2006). Estudo de alguns métodos usados para avaliar o comportamento higiênico de ecotipos locais de abelhas portuguesas. RPCV , 101, 45-49. Disponible en

http://www.fmv.ulisboa.pt/spcv/PDF/pdf6_2006/5 57_558_45_49.pdf

- Principal, J., D’Aubeterre, R., Barrios, C. et al. (2008). Comportamiento higiénico de las abejas africanizadas (Apis mellifera scutellata Lepeletier) en apiarios del estado Lara. Zootecnia Tropical, 26(2), 1667-173. Disponible en: http://www.bioline.org.br/pdf?zt08021

- Sanabria, J., Demedio, J., Pérez, T. et al. (2015). Índices de infestación por Varroa destructor en colmenas sin medidas de control. Rev. Salud Anim., 37(2), 118-124. Disponible en: http://scielo.sld.cu/pdf/rsa/v37n2/rsa07215.pdf

- Vandame, R. (2001). Control Alternativo de Varroa en Apicultura. Edición 2.2.1. p. 1-29. 